\title{
Relação da saúde bucal com reações hansênicas em município hiperendêmico para hanseníase
}

\section{Relation between oral health and hansen's disease reactions in a hyperendemic city for hansen's disease}

\author{
Adriano de Aguiar Filgueira' (D), Maria Socorro Carneiro Linhares², Mariana Ramalho Farias ${ }^{3}$, \\ Angelo Giuseppe Roncalli da Costa Oliveira ${ }^{4}$, Ana Karine Macedo Teixeira ${ }^{3}$ \\ 'Residência Multiprofissional em Saúde da Família, Universidade Estadual Vale do Acaraú (UVA) - Sobral (CE), Brasil. \\ ${ }^{2}$ Departamento de Enfermagem, Universidade Estadual Vale do Acaraú (UVA) - Sobral (CE), Brasil. \\ ${ }^{3}$ Universidade Federal do Ceará (UFC), Campus Sobral - Sobral (CE), Brasil. \\ ${ }^{4}$ Universidade Federal do Rio Grande do Norte (UFRN) - Natal (RN), Brasil.
}

Como citar: Filgueira AA, Linhares MSC, Farias MR, Oliveira AGRC, Teixeira AKM. Relação da saúde bucal com reações hansênicas em município hiperendêmico para hanseníase. Cad Saúde Colet, 2020;28(1):44-55.

https://doi.org/10.1590/1414-462X202028010033

\section{Resumo}

Introdução: Péssimas condições de saúde bucal vêm sendo apontadas como prejudiciais às pessoas acometidas pela hanseníase, pois contribuem para a piora do quadro clínico desses indivíduos. Objetivo: Este trabalho objetiva avaliar as condições de saúde bucal dos indivíduos atendidos por um serviço especializado no município de Sobral, no Ceará, e comparar dois grupos (com e sem reação hansênica) quanto às condições orais. Método: Trata-se de um estudo transversal realizado no Ambulatório de Hanseníase do município de Sobral, no Ceará, com 56 pacientes em tratamento. Foram realizados um levantamento epidemiológico em saúde bucal e uma entrevista que coletaram informações sobre condições de saúde bucal, dados socioeconômicos, utilização dos serviços odontológicos e percepção entre doença e saúde bucal. Para a análise estatística entre os grupos, foi realizada uma análise bivariada, seguida por uma análise multivariada. Resultados: Houve predomínio de sexo masculino, baixa escolaridade e diagnóstico multibacilar. Apesar da relação estatisticamente significante entre reação hansênica e cárie dentária, necessidade de exodontia e/ou endodontia, sangramento gengival, cálculo dentário e bolsa periodontal, apenas esta última apresentou associação significativa $(p=0,019)$ na análise multivariada. Conclusão: Os participantes deste estudo apresentaram precárias condições de saúde bucal, sendo piores naqueles com reações hansênicas.

Palavras-chave: hanseníase; saúde bucal; odontologia em saúde pública.

\begin{abstract}
Background: Poor oral health conditions are being considered as harmful for people affected by Hansen's disease, since they contribute to worsening the clinical condition of these individuals. Objective: This study aims to evaluate the oral health conditions of individuals attended by a specialized service in the city of Sobral, Ceará, and to compare two groups (with and without Hansen's disease reaction) regarding oral conditions. Method: A cross-sectional study was carried out at the Ambulatório de Hanseníase in the city of Sobral, Ceará, Brazil, with 56 patients being treated. An epidemiological survey was conducted on oral health and an interview that collected information on oral health conditions, socioeconomic data, use of dental services and perception between the disease and oral health. For the statistical analysis between the groups, a bivariate analysis was performed, followed by a multivariate analysis. Results: Predominance was the male gender, low level of schooling and multibacillary diagnosis. Despite the statistically significant relationship between presence of Hansen's disease reaction and the presence of dental caries, need for exodontia and / or endodontics, gingival bleeding, dental calculus and
\end{abstract}

Trabalho realizado no Ambulatório de Hanseníase, Centro de Infectologia - Sobral (CE), Brasil.

Correspondência: Adriano de Aguiar Filgueira. E-mail: adriano.odonto@yahoo.com.br

Fonte de financiamento: nenhuma.

Conflito de interesses: nada a declarar.

Recebido em: Mar. 04, 2017. Aprovado em: Jun. 04, 2019 cadernos

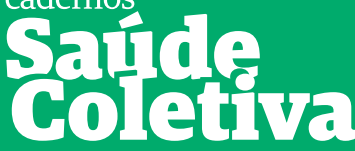

ISSN 2358-291X (Online)

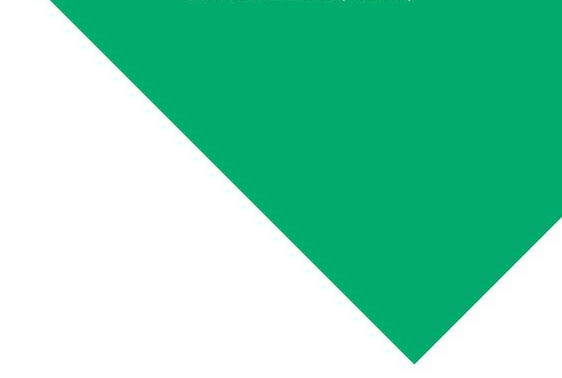


periodontal pocket, only the latter had a significant association $(p=0.019)$ in the multivariate analysis. Conclusion: The participants of this study presented poor oral health conditions, being worse in those who presented Hansen's disease reactions.

Keywords: leprosy; oral health; public health dentistry.

\section{INTRODUÇÃO}

Segundo o Ministério da Saúde ${ }^{1}$, hanseníase é uma doença crônica granulomatosa proveniente de infecção causada pelo Mycobacterium leprae. Esse bacilo afeta a pele e as células dos nervos periféricos, resultando em lesões cutâneas e neuropatia².

O estigma sobre a doença ${ }^{3-5}$ faz com que o preconceito contra os pacientes acometidos pela hanseníase aumente à medida que os sinais e sintomas se tornam mais evidentes tanto para o indivíduo infectado quanto para a sociedade. As formas multibacilares da doença são mais susceptíveis a desenvolver sequelas e/ou reações advindas da hanseníase, o que torna mais notória a infecção pelo bacilo de Hansen e, consequentemente, o aumento da discriminação $0^{3,5}$.

Quando não tratada precocemente, a hanseníase pode levar a sequelas neurológicas, oftalmológicas e motoras ${ }^{4}$. As deficiências físicas ocasionadas pelo comprometimento neurológico periférico, que podem afetar os indivíduos antes, no decorrer ou depois do tratamento, são mais frequentes nas formas mais graves da doença ${ }^{6}$.

Apesar de os episódios reacionais poderem aparecer em qualquer uma das formas clínicas, são mais raros nos casos mais brandos da doença ${ }^{7}$. Estima-se que $25-30 \%$ das pessoas com hanseníase desenvolvem reações ou dano neural em algum momento, sendo desencadeada, principalmente, por processos infecciosos, infestações, distúrbios hormonais e fatores emocionais ${ }^{8}$.

Embora poucos estudos tenham encontrado relação das infecções odontológicas com a presença de reações hansênicas, processos infecciosos em cavidade oral, assim como em todo o corpo, podem estimular ações do sistema imunológico, o que favorece o aparecimento de reações em pacientes acometidos pelo bacilo de Hansen ${ }^{9-11}$.

O diagnóstico precoce, o tratamento oportuno para todos os casos diagnosticados, a prevenção e o tratamento de incapacidades, bem como a vigilância dos contatos domiciliares, são propostas para o controle da hanseníase no Brasil de acordo com o manual técnico-operacional das Diretrizes para Vigilância, Atenção e Eliminação da Hanseníase como Problema de Saúde Pública, normatizado pela Portaria no 149, de fevereiro de 2016 ${ }^{12,13}$. Para isso, uma abordagem mais integral deve ser realizada, considerando como relevantes o momento do diagnóstico, o período de tratamento com a poliquimioterapia (PQT) e o período de pós-alta da PQT, de acordo com questões como deficiência, limitação de atividade e restrição de participação social ${ }^{10}$.

Em 2016, o Brasil perdeu em números de casos novos apenas para a Índia, registrando 25.218 ocorrências, atingindo uma taxa de detecção de 12,2/100 mil habitantes e fazendo com que o país fosse classificado como de alta carga para a doença. O país vem registrando queda no número de casos novos registrados ao longo do tempo por causa, principalmente, das estratégias do Ministério da Saúde quanto à abordagem da doença no Programa Saúde na Escola dos municípios com taxa de detecção elevada, não só para identificar casos entre as crianças, mas para informar os estudantes e mobilizar o conjunto das famílias ${ }^{14,15}$.

No Ceará, a taxa de detecção em 2017 foi de 18,7 novos casos a cada 100 mil habitantes, padrão considerado alto pelo Ministério da Saúde, segundo definido pelo manual técnico-operacional das Diretrizes para Vigilância, Atenção e Eliminação da Hanseníase como Problema de Saúde Pública ${ }^{12,13}$. O município de Sobral, situado na região noroeste do Estado do Ceará, apresenta elevadas taxas de detecção de hanseníase e foi considerado hiperendêmico por muitos anos, com taxas superiores a 40 casos novos a cada 100 mil habitantes. Em 2017, apresentou taxa de detecção de 38,7 / 100 mil habitantes, sendo classificado no parâmetro muito alto ${ }^{14,16,17}$.

Este estudo tem o objetivo de avaliar as condições de saúde bucal de indivíduos acometidos por sequelas de hanseníase e reações hansênicas atendidos no Ambulatório de 
Hanseníase de Sobral, no Ceará, comparando os dois grupos quanto às doenças bucais e ao uso dos serviços odontológicos.

A crescente associação entre saúde bucal e reação hansênica tem tornado relevantes os estudos com essa temática. Além disso, a região geográfica de realização desta pesquisa é considerada hiperendêmica para a hanseníase e não possui um protocolo definido para o atendimento odontológico desses pacientes, o que pode favorecer a implementação de ações de vigilância em saúde bucal na região.

\section{MÉTODO}

Trata-se de um estudo transversal analítico, conduzido no Ambulatório de Hanseníase localizado no município de Sobral, no Ceará. Os sujeitos da pesquisa corresponderam a 56 pacientes diagnosticados com hanseníase e que, no período de coleta de dados desta pesquisa (setembro a dezembro de 2014), apresentavam alguma sequela advinda da hanseníase ou algum tipo de reação hansênica e estavam em tratamento no serviço especializado, independentemente do tempo de contato com o serviço de referência.

A microrregião de Sobral contém 24 municípios da 11ª CRES (Coordenadoria Regional de Saúde) e um contingente populacional de quase 600 mil habitantes ${ }^{18}$. O Ambulatório de Hanseníase localizado no município de Sobral é o principal centro de referência aos pacientes com hanseníase da região, possuindo serviço médico e de fisioterapia especializados principalmente aos indivíduos que apresentam condições mais graves da doença, como reações e/ou sequelas de hanseníase.

Foram consideradas sequelas de hanseníase qualquer deforminadade/incapacidade que dificultasse a realização de atividades do dia a dia e que foram consequências da infecção pelo bacilo de Hansen, como mãos em garra, pé caído, entre outras. As reações hansênicas, por outro lado, poderiam ser do tipo 1 ou 2. Ambas as condições foram diagnosticadas por uma médica especialista e uma fisioterapeuta em conjunto. Com base nessas condições, os pacientes foram alocados em dois grupos para que fosse possível a comparação das condições de saúde bucal com o uso dos serviços odontológicos entre eles.

Foram excluídos da pesquisa aqueles que estavam realizando tratamento simultâneo para reações hansênicas e sequelas de hanseníase por causa da dificuldade de enquadramento em um dos grupos. Como critério de inclusão, visando reduzir viés de seleção, foi adotada a presença de pelo menos um dente em cavidade oral para que se pudesse avaliar a presença de infecção oral.

A coleta de dados ocorreu no Ambulatório de Hanseníase, após a consulta médica e/ou fisioterápica para tratamento do agravo da hanseníase. A coleta consistiu em duas etapas: levantamento epidemiológico e entrevista com base em um roteiro estruturado.

O levantamento epidemiológico foi fundamentado na metodologia do Projeto Saúde Bucal Brasil (SB Brasil). Foi investigada a presença de cárie dentária a partir do índice CPO-D (número de dentes cariados, perdidos e restaurados) e ceo-d (número de dentes cariados, indicados para exodontia e restaurados), doença periodontal a partir do índice IPC (índice periodontal comunitário, no qual são avaliados o sangramento gengival, a presença de cálculo dentário e a bolsa periodontal) e PIP (indicativo de perda de inserção periodontal) e índice de uso e necessidade de prótese dentária. Para o exame odontológico, foram utilizados o espelho plano bucal e a sonda periodontal, ambos esterilizados, e o procedimento foi realizado sob luz natural e com examinador e paciente sentados, conforme preconizado pela Organização Mundial de Saúde (OMS) ${ }^{19}$.

As seguintes informações foram coletadas: dados socioeconômicos (idade, sexo, escolaridade, renda familiar e emprego), condições de saúde (desde outros agravos à saúde que poderiam ocasionar respostas do sistema imunológico até condições que alteravam níveis hormonais, como puberdade e gestação), uso dos serviços odontológicos (se já foi ao dentista alguma vez na vida, última vez que procurou o serviço odontológico, tipo de serviço odontológico utilizado, se realizou e concluiu o tratamento nos últimos 12 meses), percepção do paciente em relação à doença, presença de reações hansênicas e classificação operacional. 
A coleta de dados foi realizada por um residente de Odontologia da Residência Multiprofissional em Saúde da Família do município de Sobral, devidamente capacitado e calibrado.

As variáveis foram expressas na forma de frequências absolutas. Para avaliar a existência de associação entre condições socioeconômicas, utilização do serviço odontológico e condições de saúde bucal (variáveis independentes) com a presença de reação hansênica (variável dependente), foram empregados o teste do qui-quadrado de Pearson $\left(x^{2}\right)$ e o teste exato de Fisher. Nas análises, foi considerado o nível de significância de 5\%. As informações obtidas foram analisadas pelo programa Statistical Package for the Social Sciences (SPSS), versão 20.

A Comissão Científica do município de Sobral autorizou a realização da pesquisa, sendo esta posteriormente aprovada pelo Comitê de Ética em Pesquisa da Universa Estadual Vale do Acaraú (CEP/UVA - processo no 793.452, de 10 de setembro de 2014). Foram respeitados todos os princípios éticos da Resolução n 466/2012, e todos os pacientes assinaram o Termo de Consentimento Livre e Esclarecido (TCLE).

\section{RESULTADOS}

Participaram da pesquisa 56 indivíduos que estavam em tratamento no Ambulatório de Hanseníase de Sobral no período de setembro a dezembro de 2014 , em que 50\% eram pacientes acometidos por sequelas de hanseníase e os outros $50 \%$ estavam em tratamento para reações hansênicas. Além disso, $66 \%$ haviam concluído o tratamento poliquimioterápico para a hanseníase. A classificação operacional multibacilar correspondeu a $85,7 \%$ dos participantes. Um percentual de $38,3 \%$ apontou que estava passando ou passou recentemente por alguma das condições de saúde investigadas, dos quais $81,8 \%$ afirmaram ter hipertensão e/ou diabetes. Puberdade, gravidez, puerpério/amamentação e cirurgia recente não foram relatadas pelos participantes da pesquisa.

Na Tabela 1, são representados os dados socioeconômicos, o uso dos serviços odontológicos e a percepção dos pacientes em relação à doença. Dos participantes da pesquisa, $67,9 \%$ eram do sexo masculino. A faixa etária de adulto jovem e adulto (de 20 a 59 anos) representou a maior parcela dos indivíduos, com $69,6 \%$, bem como aqueles que declararam possuir até 7 anos de estudo, com 60,7\%, e renda familiar de até 1 salário mínimo, com 35,7\%, sendo o valor máximo desta de 2,5 salários mínimos.

Tabela 1. Distribuição dos pacientes com hanseníase segundo perfil socioeconômico, utilização dos serviços odontológicos e compreensão da doença em relação à saúde bucal, Sobral, 2014

\begin{tabular}{|c|c|c|}
\hline & $\mathbf{n}$ & $\%$ \\
\hline \multicolumn{3}{|l|}{ Sexo } \\
\hline Masculino & 38 & 67,9 \\
\hline Feminino & 18 & 32,1 \\
\hline \multicolumn{3}{|l|}{ Idade } \\
\hline Até 9 anos & 1 & 1,8 \\
\hline 10-19 anos & 4 & 7,1 \\
\hline 20-59 anos & 39 & 69,6 \\
\hline 60 anos ou mais & 12 & 21,4 \\
\hline \multicolumn{3}{|l|}{ Escolaridade } \\
\hline Até 7 anos & 39 & 69,6 \\
\hline 8 anos ou mais & 17 & 30,4 \\
\hline
\end{tabular}

n: número de pessoas 
Tabela 1. Continuação...

\begin{tabular}{|c|c|c|}
\hline VARIÁVEL & $\mathbf{n}$ & $\%$ \\
\hline \multicolumn{3}{|l|}{ Trabalha } \\
\hline Sim & 34 & 60,7 \\
\hline Não & 22 & 39,3 \\
\hline \multicolumn{3}{|l|}{ Renda familiar } \\
\hline Até 1 salário mínimo & 20 & 35,7 \\
\hline Acima de 1 salário mínimo & 36 & 64,3 \\
\hline \multicolumn{3}{|l|}{ Classificação operacional } \\
\hline Paucibacilar & 8 & 14,3 \\
\hline Multibacilar & 48 & 85,7 \\
\hline \multicolumn{3}{|l|}{ Ida ao dentista alguma vez na vida } \\
\hline Sim & 49 & 87,5 \\
\hline Não & 7 & 12,5 \\
\hline \multicolumn{3}{|l|}{ Última vez que foi ao dentista } \\
\hline Menos de 1 ano & 23 & 46,9 \\
\hline 1 ano ou mais & 26 & 53,1 \\
\hline \multicolumn{3}{|l|}{ Onde foi ao dentista pela última vez } \\
\hline Serviço público & 30 & 61,2 \\
\hline Serviço privado & 19 & 38,8 \\
\hline \multicolumn{3}{|l|}{ Hanseníase traz prejuízo para a saúde bucal } \\
\hline Sim & 16 & 28,6 \\
\hline Não & 38 & 67,9 \\
\hline Não soube responder & 2 & 3,6 \\
\hline \multicolumn{3}{|l|}{ Dificuldade para realizar higiene bucal } \\
\hline Sim & 15 & 26,8 \\
\hline Não & 41 & 73,2 \\
\hline \multicolumn{3}{|l|}{ Orientação para procurar o serviço odontológico } \\
\hline Sim & 22 & 39,3 \\
\hline Não & 34 & 60,7 \\
\hline \multicolumn{3}{|c|}{ Conclusão de tratamento odontológico nos últimos 12 meses } \\
\hline Sim & 11 & 47,8 \\
\hline Não & 12 & 52,2 \\
\hline \multicolumn{3}{|l|}{ Última visita ao dentista } \\
\hline Antes do tratamento da hanseníase & 20 & 35,7 \\
\hline Durante ou após o tratamento da hanseníase & 29 & 51,8 \\
\hline Nunca foi ao dentista & 7 & 12,5 \\
\hline
\end{tabular}

$\mathrm{n}$ : número de pessoas 
Um percentual de $12,5 \%$ relatou nunca ter ido ao dentista em algum momento da vida. Dos que frequentaram o dentista pelo menos uma vez na vida, $61,2 \%$ procuraram o serviço público. Com relação à última vez que foram ao serviço odontológico, 46,9\% disseram ter ido há menos de um ano.

Ademais, 67,9\% relataram não perceber algum prejuízo à saúde bucal advindo da hanseníase. No entanto, $26,8 \%$ dos indivíduos apontaram ter dificuldades para realizar a higiene oral, normalmente em razão das sequelas deixadas pela doença nos membros superiores.

Ainda, 60,7\% afirmaram que não houve orientação para a procura do serviço odontológico no início do tratamento para a hanseníase, e 51,8\% procuraram o dentista pela última vez durante a realização ou após o término do tratamento farmacológico da hanseníase. Dos indivíduos que realizaram tratamento odontológico nos últimos 12 meses, 47,8\% conseguiram fazer tratamento completo.

Na Tabela 2, constam a média do índice CPO-D e seus componentes segundo a faixa etária. Verificou-se o aumento do índice com a idade: enquanto o CPO-D foi de 4,00 na faixa etária de 10 a 19 anos, alcançou 18,08 para aqueles com idade maior ou igual a 60 anos. A exceção foi uma criança de 6 anos que relatou nunca ter ido ao dentista e apresentou índice de ceo-d igual a 13, correspondendo exclusivamente ao componente cariado. De 10 a 19 anos, o componente "dente restaurado" foi o que prevaleceu, enquanto, nas idades mais elevadas, o componente "dente perdido" foi o predominante.

Tabela 2. Média do índice CPO-D e proporção de seus componentes segundo faixa etária, Sobral, 2014

\begin{tabular}{lcccccc} 
Faixa etária & $\mathbf{n}$ & $\begin{array}{c}\text { Cariado } \\
\text { (SD) }\end{array}$ & $\begin{array}{c}\text { Restaurado } \\
\text { com cárie } \\
\text { (SD) }\end{array}$ & $\begin{array}{c}\text { Restaurado } \\
\text { sem cárie } \\
\text { (SD) }\end{array}$ & $\begin{array}{c}\text { Perdido } \\
\text { (SD) }\end{array}$ & CPOD (SD) \\
\hline $10-19$ anos & 4 & $1,25(1,5)$ & 0 & $3,75(2,8)$ & $0,25(0,5)$ & $4,00(3,2)$ \\
\hline $20-59$ anos & 39 & $2,95(4,3)$ & $0,10(0,3)$ & $1,31(2,7)$ & $8,05(7,4)$ & $9,46(7,0)$ \\
\hline 60 anos ou mais & 12 & $2,08(2,9)$ & 0 & $0,50(1,7)$ & $17,58(7,1)$ & $18.08(6,8)$ \\
\hline
\end{tabular}

n: número de pessoas; SD: desvio padrão

Na Tabela 3, constam os resultados relacionados à condição do periodonto. Na faixa etária de 10 a 19 anos, metade apresentou sangramento gengival à sondagem, com presença de cálculo dentário. Nas demais faixas etárias, a existência de cálculo foi a condição periodontal mais prevalente, seguida de sangramento gengival e bolsa periodontal.

Tabela 3. Frequência de indivíduos segundo o sextante com pior condição periodontal e faixa etária, Sobral, $2014^{*}$

\begin{tabular}{|c|c|c|c|c|c|c|c|}
\hline \multirow{2}{*}{ Faixa etária } & \multirow{2}{*}{$\mathbf{n}$} & \multicolumn{2}{|c|}{ Sangramento } & \multicolumn{2}{|c|}{ Cálculo } & \multicolumn{2}{|c|}{ Bolsa periodontal } \\
\hline & & $\mathbf{n}$ & $\%$ & $\mathbf{n}$ & $\%$ & $\mathbf{n}$ & $\%$ \\
\hline 10-19 anos & 4 & 2 & 50 & 2 & 50 & 0 & 0 \\
\hline 20-59 anos & 39 & 29 & 74,3 & 36 & 92,3 & 21 & 53,8 \\
\hline 60 anos ou mais & 12 & 9 & 75 & 10 & 83,3 & 5 & 41,6 \\
\hline
\end{tabular}

*Um mesmo indivíduo pode apresentar mais de uma condição periodontal; n: número de pessoas

Com relação ao uso e à necessidade de prótese dentária, verificou-se que $75 \%$ dos pacientes não faziam uso de prótese superior, apesar de $46,4 \%$ necessitarem de algum tipo de prótese na arcada superior, sendo 5,4\% de prótese total (PT) e 41\% de prótese parcial removível (PPR). Dos $25 \%$ que faziam uso de prótese superior, $7,1 \%$ eram PT, $16,1 \%$, PPR, e 1,8\%, ponte fixa. Quanto à prótese inferior, $92,9 \%$ não faziam uso, porém $73,2 \%$ possuíam a necessidade dela, sendo $3,6 \%$ de PT e $69,7 \%$ de PPR. Dos 7,2\% em uso de prótese inferior, todos eram de prótese parcial removível. 
Na Tabela 4, em análise não ajustada, não houve associação estatisticamente significativa entre presença de reação hansênica e condições de saúde, estresse, sexo, renda familiar, escolaridade e última vez que procurou um cirurgião-dentista. Já em relação às condições de saúde bucal, presença de pelo menos um dente com cárie $(p=0,007)$, necessidade de exodontia/endodontia em pelo menos um dente $(p=0,004)$, presença de pelo menos um sextante com sangramento gengival $(p=0,024)$, cálculo dentário $(p=0,002)$ e bolsa periodontal $(p=0,001)$ apresentaram associação estatística significativa com a presença de reação hansênica.

Tabela 4. Distribuição dos pacientes com reação hansênica segundo variáveis sociodemográficas, uso de serviço odontológico e condições de saúde bucal, Sobral, 2014

\begin{tabular}{|c|c|c|c|c|c|c|c|c|}
\hline \multirow{3}{*}{ Variável } & \multicolumn{4}{|c|}{ Reação hansênica } & \multicolumn{2}{|c|}{ Não ajustada } & \multicolumn{2}{|c|}{ Ajustada } \\
\hline & \multicolumn{2}{|c|}{ Sim } & \multicolumn{2}{|c|}{ Não } & \multirow{2}{*}{$\mathbf{p}$} & \multirow{2}{*}{$\mathrm{RP}(\mathrm{IC})$} & \multirow{2}{*}{$\mathbf{p}$} & \multirow{2}{*}{$\mathbf{R P}(\mathrm{IC})$} \\
\hline & $\mathbf{n}$ & $\%$ & $\mathbf{n}$ & $\%$ & & & & \\
\hline \multicolumn{9}{|l|}{ Sexo } \\
\hline Masculino & 18 & 47,4 & 20 & 52,6 & \multirow{2}{*}{0,55} & \multirow{2}{*}{$\begin{array}{c}1,17 \\
(0,68-1,99)\end{array}$} & \multirow{2}{*}{ - } & \multirow{2}{*}{-} \\
\hline Feminino & 10 & 55,6 & 8 & 44,4 & & & & \\
\hline \multicolumn{9}{|l|}{ Renda } \\
\hline Até 1 salário mínimo & 7 & 35,0 & 13 & 65,0 & \multirow{2}{*}{0,12} & \multirow{2}{*}{$\begin{array}{c}0,6 \\
(0,31-1,15)\end{array}$} & \multirow{2}{*}{ - } & \multirow{2}{*}{-} \\
\hline Mais que 1 salário mínimo & 21 & 58,3 & 15 & 41,7 & & & & \\
\hline \multicolumn{9}{|l|}{ Escolaridade } \\
\hline Até 7 anos & 18 & 46,2 & 21 & 53,8 & \multirow{2}{*}{0,36} & \multirow{2}{*}{$\begin{array}{c}1,27 \\
(0,75-2,14)\end{array}$} & \multirow{2}{*}{-} & \multirow{2}{*}{-} \\
\hline 8 anos ou mais & 10 & 58,8 & 7 & 41,2 & & & & \\
\hline \multicolumn{9}{|l|}{$\begin{array}{l}\text { Última vez que procurou } \\
\text { um cirurgião-dentista }\end{array}$} \\
\hline $\begin{array}{l}\text { Antes do tratamento da } \\
\text { hanseníase }\end{array}$ & 10 & 50,0 & 10 & 50,0 & \multirow{2}{*}{0,71} & \multirow{2}{*}{$\begin{array}{c}0,89 \\
(0,49-1,62)\end{array}$} & \multirow{2}{*}{ - } & \multirow{2}{*}{-} \\
\hline $\begin{array}{l}\text { No decorrer ou depois do } \\
\text { tratamento da hanseníase }\end{array}$ & 13 & 44,8 & 16 & 55,2 & & & & \\
\hline \multicolumn{9}{|l|}{ Estresse } \\
\hline Sim & 6 & 66,7 & 3 & 33,3 & $0,21 *$ & $\begin{array}{c}1,42 \\
(0,81-2,47)\end{array}$ & - & - \\
\hline Não & 22 & 46,8 & 25 & 53,2 & & & & \\
\hline \multicolumn{9}{|l|}{ Condições de saúde } \\
\hline Sim & 10 & 45,5 & 12 & 54,5 & 0,591 & $\begin{array}{c}0,85 \\
(0,49-1,49)\end{array}$ & - & - \\
\hline Não & 18 & 52,9 & 16 & 47,1 & & & & \\
\hline \multicolumn{9}{|l|}{ Cárie } \\
\hline Sim & 22 & 68,8 & 10 & 31,2 & 0007 & 2,75 & 0,085 & 2,06 \\
\hline Não & 6 & 25,0 & 18 & 75,0 & & $(1,32-5,71)$ & & $(0,90-4,70)$ \\
\hline
\end{tabular}

*Teste exato de Fisher; $n$ : número de pessoas; $p$ : valor de p; RP (IC): razão de prevalência (intervalo de confiança) 
Tabela 4. Continuação...

\begin{tabular}{|c|c|c|c|c|c|c|c|c|}
\hline \multirow{3}{*}{ Variável } & \multicolumn{4}{|c|}{ Reação hansênica } & \multicolumn{2}{|c|}{ Não ajustada } & \multicolumn{2}{|c|}{ Ajustada } \\
\hline & \multicolumn{2}{|c|}{ Sim } & \multicolumn{2}{|c|}{ Não } & \multirow{2}{*}{$\mathbf{p}$} & \multirow{2}{*}{$\mathrm{RP}(\mathrm{IC})$} & \multirow{2}{*}{$\mathbf{p}$} & \multirow{2}{*}{ RP (IC) } \\
\hline & $\mathbf{n}$ & $\%$ & $n$ & $\%$ & & & & \\
\hline \multicolumn{9}{|c|}{$\begin{array}{l}\text { Necessidade de exodontia } \\
\text { e/ou endodontia }\end{array}$} \\
\hline $\operatorname{Sim}$ & 15 & 75,0 & 5 & 25,0 & \multirow{2}{*}{0,004} & \multirow{2}{*}{$\begin{array}{c}2,07 \\
(1,25-3,43)\end{array}$} & \multirow{2}{*}{0,626} & \multirow{2}{*}{$\begin{array}{c}0,87 \\
(0,49-1,52)\end{array}$} \\
\hline Não & 13 & 36,1 & 23 & 63,9 & & & & \\
\hline \multicolumn{9}{|l|}{ Sangramento } \\
\hline Sim & 25 & 62.5 & 15 & 37,5 & \multirow{2}{*}{$0,024^{*}$} & \multirow{2}{*}{$\begin{array}{c}3,33 \\
(1,16-9,50)\end{array}$} & \multirow{2}{*}{-} & \multirow{2}{*}{-} \\
\hline Não & 3 & 18,8 & 13 & 81,2 & & & & \\
\hline \multicolumn{9}{|l|}{ Cálculo } \\
\hline Sim & 28 & 58,3 & 20 & 41,7 & \multirow{2}{*}{$0,002^{*}$} & \multirow{2}{*}{$\begin{array}{c}2,40 \\
(1,71-3,35)\end{array}$} & \multirow{2}{*}{ - } & \multirow{2}{*}{-} \\
\hline Não & 0 & 0,0 & 8 & 100,0 & & & & \\
\hline \multicolumn{9}{|l|}{ Bolsa periodontal } \\
\hline $\operatorname{Sim}$ & 20 & 79,6 & 6 & 23,1 & \multirow{2}{*}{0,001} & \multirow{2}{*}{$\begin{array}{c}2,88 \\
(1,53-5,41)\end{array}$} & \multirow{2}{*}{0,019} & \multirow{2}{*}{$\begin{array}{c}2,33 \\
(1,14-4,75)\end{array}$} \\
\hline Não & 8 & 26,7 & 22 & 73,3 & & & & \\
\hline
\end{tabular}

*Teste exato de Fisher; $n$ : número de pessoas; p: valor de p; RP (IC): razão de prevalência (intervalo de confiança)

$\mathrm{Na}$ análise ajustada do mesmo modelo, todas as variáveis relacionadas às condições de saúde, com exceção de sangramento e cálculo dentário (utilizado o teste de Fisher por causa da pequena amostra), apresentaram relação estatisticamente significante, porém apenas a presença de bolsa periodontal permaneceu com associação significativa $(p=0,019)$.

\section{DISCUSSÃO}

Os pacientes que participaram do estudo estavam em tratamento no Ambulatório de Hanseníase de Sobral para reações ou sequelas advindas da hanseníase. A pequena amostra de 56 indivíduos pode ser justificada pela dificuldade de enquadramento deles em apenas um dos grupos: com ou sem reação hansênica, bem como pelo número total de pacientes desdentados que foram excluídos da pesquisa. Além disso, o curto período de coleta de dados e a pequena demanda de pacientes novos no serviço (os mesmos pacientes retornavam várias vezes para continuidade das sessões de fisioterapia e/ou reavaliações médicas) contribuíram para o baixo número de pacientes avaliados.

Estudos apontam que as reações e as sequelas podem ser detectadas antes, no decorrer ou depois do tratamento poliquimioterápico da doença ${ }^{7,8}$. Como a medicação para o tratamento da hanseníase tem início logo após o diagnóstico por um profissional habilitado, o que era condição essencial para a inclusão nesta pesquisa, então todos os pacientes deste estudo haviam iniciado e/ou concluído o tratamento poliquimioterápico.

Dos indivíduos participantes, a maioria foi acometida pelas formas mais graves da doença, o que condiz com a literatura quando aponta que os pacientes mais propícios a desenvolver reações e/ou apresentar alguma sequela da hanseníase são aqueles que apresentam a forma multibacilar, pois possuem alto teor bacilífero ${ }^{2,20,21}$.

Alguns estudos também encontraram uma maior frequência do sexo masculino nos pacientes infectados pelo Mycobacterium leprae ${ }^{9,21}$. O percentual de homens aumenta quando se trata das formas mais graves de hanseníase ${ }^{2}$. A explicação de os homens serem os mais acometidos pelas formas mais graves da doença se dá pela dificuldade histórica de reconhecer 
suas necessidades, cultivando o pensamento mágico que rejeita a possibilidade de adoecer. A Política Nacional de Atenção Integral à Saúde do Homem afirma que eles adentram no sistema de saúde por meio da atenção especializada, o que leva ao agravo da morbidade pelo retardamento da atenção, gerando maior custo ao SUS ${ }^{22}$.

A faixa etária mais prevalente nesta pesquisa foi a de adulto jovem e adulto ( 20 a 59 anos). Outros estudos também encontraram a idade adulta como a mais prevalente ${ }^{11,23}$. Capelo e Pagliuca ${ }^{24}$ verificaram que há uma maior tendência das formas multibacilares com o avançar da idade e que a maior parcela dos pacientes com hanseníase está na faixa etária economicamente ativa, o que pode dificultar o diagnóstico, tratamento e acompanhamento desses indivíduos a fim de reduzir o aparecimento de sequelas e as reações advindas da hanseníase ${ }^{24}$.

A baixa renda familiar e a escolaridade deficiente são evidências constantes nas pesquisas que abordam pacientes com hanseníase. Estudos apontam uma prevalência de 65 a $69,6 \%$ de pacientes com escolaridade inferior a 8 anos. Quanto à renda familiar, a quantia de até 2 salários mínimos está presente entre 73,8 a $89 \%$ das famílias que possuem pelo menos um indivíduo acometido pelo Mycobacterium leprae $e^{9,11,14}$.

No que se refere à percepção de possíveis prejuízos à cavidade oral advindos da hanseníase, quase $68 \%$ dos pacientes disseram não perceber relação, valor que se aproximou de outros estudos que tiveram percentual variando de 64 a $76 \%^{11,25}$. Um estudo realizado por Almeida et. al. observou uma associação entre a necessidade de tratamento odontológico e a escolaridade do paciente, em que um maior grau de escolaridade favoreceu a percepção da pessoa quanto à necessidade de tratamento ${ }^{9}$. A baixa escolaridade pode justificar, portanto, a não percepção de possíveis prejuízos à cavidade oral por não conferir a devida importância à saúde bucal.

Uma pesquisa realizada em Fortaleza abordando pacientes com hanseníase verificou que $98 \%$ realizaram tratamento odontológico pelo menos uma vez na vida ${ }^{25}$. Neste estudo, o percentual de pacientes que frequentaram um dentista em algum momento da vida ficou em torno de $87,5 \%$, número que se aproxima de outro estudo também realizado no município de Sobral, cujo número ficou em aproximadamente $89 \%{ }^{11}$. Dados do Suplemento de Saúde da Pesquisa Nacional por Amostra de Domicílios (PNAD) de 2003 mostram que 28 milhões de brasileiros (15,9\% da população) nunca foram ao dentista e que o percentual era maior nas áreas rurais, no sexo masculino e na população com até 1 salário mínimo ${ }^{18}$. Apesar do número crescente de equipes de saúde bucal (ESB) no país, o acesso aos serviços odontológicos ainda é algo deficiente ${ }^{19}$. Além disso, o desconhecimento da relação entre hanseníase e saúde bucal pelos cirurgiões-dentistas é um fator relevante para a falta de inclusão desses pacientes nas ações e assistências odontológicas. Almeida e colaboradores ${ }^{25}$ ) observaram em seus estudos que mais de $64 \%$ dos odontólogos nunca suspeitaram de algum caso da doença e que mais de $70 \%$ deles afirmaram desconhecer a relação entre hanseníase e saúde bucal.

Estudos realizados com pacientes diagnosticados com hanseníase encontraram valores aproximados sobre dificuldade de higienizar a cavidade oral por causa de problemas relacionados à hanseníase (valores variando de 13 a 15,3\%) ${ }^{25}$. Neste estudo, foi verificado percentual mais elevado (26,8\%), que pode ser justificado em razão de os sujeitos da pesquisa serem pacientes acometidos por formas mais graves da doença e em tratamento de problemas advindos da hanseníase (reações e sequelas hansênicas). Para Costa et al. ${ }^{26}$, entre os motivos que podem dificultar a higiene estão as reações que comprometem a saúde sistêmica do paciente, tornando mais difíceis os hábitos de higienização corporal, incluindo a bucal, e as sequelas da doença, por exemplo, as mãos em garra e amputações de dedos, que podem interferir diretamente na saúde da boca ${ }^{26}$.

Com relação à última vez que procurou o serviço saúde bucal, pouco mais da metade afirmou ter procurado o serviço durante ou após o tratamento da hanseníase, o que leva a crer que o fato de buscarem tratamento odontológico se deva à presença de focos de infecção em cavidade oral que podem ter levado ao agravamento da doença. Estudo realizado no mesmo município encontrou resultado diferente, relatando um percentual de $61,5 \%$ para aqueles que procuraram o serviço odontológico pela última vez antes do tratamento da hanseníase, encontrando associação estatisticamente significativa entre essa variável e a presença de 
reações hansênicas ${ }^{11}$. Na pesquisa realizada por Filgueira e colaboradores, a procura do serviço antes pode ter reduzido os focos de infecção oral, levando ao não aparecimento de reações.

Apesar de a hanseníase ser uma das prioridades do Ministério da Saúde no Brasil e estar entre as doenças negligenciadas e em eliminação que devem ser priorizadas pelos serviços de saúde, a Odontologia ainda deixa a desejar no acesso aos serviços de saúde bucal pelos pacientes com hanseníase. Além disso, é rara a continuidade do tratamento odontológico, fato que pode ser tanto pela falta de abertura da Odontologia na priorização desses pacientes quanto pela resistência destes a realizar o tratamento odontológico ${ }^{27}$.

Com o objetivo de superar o modelo biomédico de atenção às doenças, a Política Nacional de Saúde Bucal propõe duas formas de inserção transversal da saúde bucal nos diferentes programas integrais de saúde: por linhas de cuidado e por condição de vida. Esta última compreende saúde da mulher, saúde do trabalhador, portadores de necessidades especiais, hipertensos, diabéticos, entre outros. A hanseníase, sendo uma doença negligenciada e em eliminação, é considerada como uma das prioridades pelo Ministério da Saúde e deve ser, portanto, um dos focos de atenção à saúde bucal por condição de vida, visto que precárias condições bucais vêm sendo apontadas como uma das causas para a piora da qualidade de vida dos pacientes acometidos pela hanseníase ${ }^{28}$.

Pacientes com hanseníase apresentam condições precárias de saúde bucal ${ }^{10}$. Os dados de cárie dentária, uso e necessidade de prótese dental em portadores de hanseníase apresentaram semelhanças com os resultados encontrados em outros estudos tanto na população geral ${ }^{19}$ quanto na população hansênica" ${ }^{11}$. As pesquisas verificaram aumento do CPO-D com o avançar da idade e elevada perda dentária na população idosa.

Quanto à condição periodontal, foram encontradas semelhanças com a população geral indicada pelo estudo SB Brasil. Ambos apontaram o cálculo dental como o mais prevalente dentre as condições do periodonto: enquanto, no SB Brasil, o percentual variou de 24 a $64,1 \%{ }^{19}$, esta pesquisa encontrou percentagem que variou de 50 a $92,3 \%$.

A condição de saúde bucal precária dos pacientes com hanseníase foi relatada em alguns estudos que defenderam a priorização dos indivíduos acometidos pelo bacilo de Hansen na atenção odontológica, de modo que o encaminhamento ao cirurgião-dentista (CD) deve ser realizado tão logo ocorra a confirmação diagnóstica. Todavia, as pesquisas também ressaltaram que a ampliação das ações do cirurgião-dentista depende da integração aos programas de capacitação ${ }^{9,10}$. Atualmente, existe uma tendência a se modificar o fazer da Odontologia dentro do SUS por meio de mudanças nas grades curriculares das universidades, inserção dos acadêmicos de Odontologia dentro de programas como o pró-pet-saúde, além dos programas de pós-graduação como as residências multiprofissionais em saúde da família, saúde coletiva e saúde pública, que visam à formação de cirurgiões-dentistas aptos a desenvolver trabalhos em equipes multiprofissionais, ampliando a integralidade e melhorando a qualidade dos serviços públicos de saúde.

Apesar da maior tendência da ocorrência de reações hansênicas quando há presença de infecções dentoalveolares, somente a presença de bolsa periodontal apresentou-se significantemente associada. Souza et al. ${ }^{29}$ acompanharam pacientes com hanseníase por três anos e verificaram associação estatisticamente significativa entre doença periodontal e surto reacional hansênico ${ }^{29}$.

A literatura aponta processos infecciosos, infecções, distúrbios hormonais e fatores emocionais como fatores desencadeantes de episódios reacionais ${ }^{7,8}$, pois ocasionam uma queda no sistema imunológico. A maioria dos pacientes que citaram estar passando por um período de estresse também estava sendo acometida por reações hansênicas, porém não se conseguiu estabelecer associação significativa. Tal fato pode ser explicado por essa condição ser autocitada pelos próprios pacientes, e alguns sujeitos da pesquisa podem ter omitido a condição, o que pode ser uma limitação do estudo.

Não foi encontrada relação significante também com problemas de saúde e presença de episódios reacionais. Apesar de 39,3\% relatarem algum problema de saúde como hipertensão e diabetes, todos apontaram fazer uso de medicação, o que pode compensar os desequilíbrios advindos dos problemas de saúde e, consequentemente, não influenciar o aparecimento das 
reações hansênicas. Além disso, a condição de saúde foi autorrelatada pelos próprios sujeitos da pesquisa, pois não foi realizada análise de prontuário, fato que representa uma limitação do estudo. Futuras pesquisas podem realizar aferição da pressão arterial e glicemia no momento da pesquisa para avaliar se existe algum problema de saúde ainda desconhecido pelo paciente acometido pelo Mycobacterium leprae.

Os pacientes acometidos pelo bacilo de Hansen em tratamento no Ambulatório de Hanseníase de Sobral apresentaram precárias condições de saúde bucal. Aqueles que tiveram reações hansênicas possuíam condições orais piores do que aqueles que realizavam tratamento apenas para sequelas, o que aponta que problemas bucais, principalmente a presença de bolsa periodontal, podem estar associados com o aparecimento de reações. Outros fatores, no entanto, como condições de saúde, estresse, renda e escolaridade não apresentaram associação com reação, mas podem ter efeito cumulativo com os problemas bucais para o aparecimento de episódios reacionais.

Os resultados apontam para uma real necessidade desses pacientes de realizar tratamento odontológico a fim de reduzir os focos de infecção em cavidade oral, diminuindo o aparecimento dos episódios reacionais e, consequentemente, melhorando as condições de vida desses indivíduos, além de evitar uma maior demanda nos serviços de atenção secundária especializados em hanseníase. O cuidado deve ser longitudinal e de forma integral, visto que mesmo aqueles que receberam alta da terapia medicamentosa podem ser acometidos tanto por sequelas quanto por reações hansênicas, sendo de extrema importância o acompanhamento de todos os profissionais da Estratégia Saúde da Família.

\section{REFERÊNCIAS}

1. Brasil. Ministério da Saúde. Doenças infecciosas e parasitárias: guia de bolso. 8. ed. Brasília; 2010.

2. Oliveira DT, Sherlock J, Melo EV, Rollemberg KCV, PaixãoTRC, AbuawadYG, et al. Clinical variables associated with leprosy reactions e and persistence of physical impairment. Rev Soc Bras Med Trop. 2013;46(5):600-4. http://dx.doi.org/10.1590/0037-8682-0100-2013. PMid:24270251.

3. Monteiro SCL. Hanseníase: políticas públicas e qualidade de vida de pacientes e seus cuidadores [dissertação]. Belo Horizonte: Fundação Oswaldo Crusz; 2010.

4. Figueiredo APP. Hanseníase: do isolamento familiar ao social [dissertação]. Gurupi: Fundação UNIRG; 2012.

5. Dalillo D, Simões MJS. Estudo do comportamento social dos pacientes de hanseníase do município de São Carlos - SP. Revista Espaço para a Saúde. 2005;7(1):10-5.

6. Nardi SMT, Paschoal VDA, Chiaravalloti-Neto F, Zanetta DMT. Deficiências após alta medicamentosa da hanseníase: prevalência e distribuição especial. Rev Saude Publica. 2012;46(6):969-77. http://dx.doi. org/10.1590/S0034-89102013005000002. PMid:23358621.

7. Foss NT, Souza CS, Goulart IMB, Gonçalvez HS, Virmond M. Hanseníase: episódios reacionais. São Paulo: Associação Médica Brasileira e Conselho Federal de Medicina; 2003. http://dx.doi.org/10.11606/issn.21767262.v36i2/4p453-459.

8. Belo Horizonte. Secretaria do Estado de Saúde de Minas Gerais. Como tratar e reconhecer reações hansênicas. Belo Horizonte; 2005. 85 p.

9. Almeida JRS. Atenção à saúde bucal dos portadores de hanseníase no município de Fortaleza-CE, Brasil [dissertação]. Fortaleza: Universidade Federal do Ceará; 2010.

10. Cortela DCB, Ignotti E. Lesões visíveis na hanseníase: o papel do cirurgião-dentista na suspeita de casos novos. Rev Bras Epidemiol. 2008;11(4):619-32. http://dx.doi.org/10.1590/S1415-790X2008000400010.

11. Filgueira AA, Paresque MAC, Carneiro SMF, Teixeira AKM. Saúde bucal em indivíduos com hanseníase no município de Sobral, Ceará. Epidemiol Serv Saúde. 2014;23(1):155-164.

12. Brasil. Ministério da Saúde. Secretaria de Vigilância em Saúde. Departamento de Vigilância das Doenças Transmissíveis. Diretrizes para vigilância, atenção e eliminação da Hanseníase como problema de saúde pública: Manual técnico-operacional. Brasília; 2016.

13. Brasil. Ministério da Saúde. Portaria no 149 de 3 de fevereiro de 2016. Legislação em Saúde. Diário Oficial da União [Internet], Brasília, DF, 3 de fevereiro de 2016 [citado em 2019 Fev 5]. Disponível em: http:// bvsms.saude.gov.br/bvs/saudelegis/gm/2016/prt0149_04_02_2016.html 
14. Brasil. Ministério da Saúde. Secretaria de Vigilância em Saúde. Hanseníase. Boletim Epidemiológico. 2018;49(4):1-10.

15. Brasil. Ministério da Saúde. Brasil quer eliminar a hanseníase até 2015 [Internet]. 2012 [citado 2014 Dez 5]. Disponível em: http://www.brasil.gov.br/saude/2012/06/brasil-quer-eliminar-a-hanseniase-ate-2015

16. Taheri JB, Mortazavi H, Moshfeghi M, Bakhshi M, Bakhtiari S, Azari-Marhabi S, et al. Oral-facial manifestations of 100 leprosy patients. Med Oral Patol Oral Cir Bucal. 2012;15(5):e728-32. http://dx.doi.org/10.4317/ medoral.17718. PMid:22322502.

17. Ceará. Secretaria de Saúde do Governo do Estado do Ceará. Informe epidemiológico hanseníase [Internet] Fortaleza: Secretaria de Saúde; 2014 [citado em 2015 Mar 19]. Disponível em: http://www.saude.ce.gov br/index.php/boletins?download=813\%3Ahanseniase-janeiro-de-2012.

18. Instituto Brasileiro de Geografia e Estatística. Pesquisa nacional por amostra de domicílios [Internet]. Rio de Janeiro: IBGE; 2013 [citado em 2014 Dez 5]. Disponível em: http://www.ibge.gov.br/home/estatistica/ pesquisas/pesquisa_resultados.php?id_pesquisa $=40$

19. Brasil. Ministério da Saúde. Departamento de Atenção Básica. Área Técnica de Saúde Bucal. Projeto SB2010: condições de saúde bucal da população brasileira no ano 2010: manual do examinador. Brasília: Ministério da Saúde; 2010.

20. Souza LWF. Reações hansênicas em pacientes em alta por cura pela poliquimioterapia. Rev Soc Bras Med Trop. 2010 nov-dez;43(6):737-9. http://dx.doi.org/10.1590/S0037-86822010000600029. PMid:21181036.

21. Pereira RMS, Silva TSO, Silva LS, Santos TC, Falcão CAM, Pinto LSS. Oral and dental condition in leprosy. Braz J Oral Sci. 2013 dez;12(4):330-4. http://dx.doi.org/10.1590/S1677-32252013000400010.

22. Brasil. Ministério da Saúde. Política Nacional de Atenção Integral à Saúde do Homem: princípios e diretrizes. Brasília: Ministério da Saúde; 2008.

23. Teixeira MAG, Silveira VM, França ER. Características epidemiológicas e clínicas em indivíduos paucibacilares e multibacilares, atendidos em dois centros de referência para hanseníase, na cidade de Recife, Estado de Pernambuco. Rev Soc Bras Med Trop. 2010 maio-jun;43(3):287-92. http://dx.doi.org/10.1590/S003786822010000300015 . PMid:20563498.

24. Capelo SC, Pagliuca LMF. Correlação das formas clínicas de hanseníase com sexo e idade. Rev Rene. 2000;1(2):16-20.

25. Almeida JRS, Alencar CHM, Barbosa JC, Dias AA, Almeida MEL. Autopercepção de pessoas acometidas pela hanseníase sobre sua saúde bucal e necessidade de tratamento. Cien Saude Colet. 2013;18(3):81726. http://dx.doi.org/10.1590/S1413-81232013000300027. PMid:23546208.

26. Costa APF, Nery JAC, Oliveira MLW, Cuzzi T, Silva MR. Oral lesions in leprosy. Indian J Dermatol Venereol Leprol. 2003;69(6):381-5. PMid:17642946.

27. Brasil. Ministério da Saúde. Secretaria de Atenção à Saúde. Departamento de Atenção Básica. Política nacional de atenção básica. Brasília: Ministério da Saúde; 2017.

28. Brasil. Ministério da Saúde. Secretaria de Atenção à Saúde. Departamento de Atenção Básica. Política nacional de saúde bucal. Brasília: Ministério da Saúde; 2004.

29. Souza VA, Emmerich A, Coutinho EM, Freitas MG, Silva EH, Merçon FG, et al. Dental and oral condition in leprosy patients from Serra, Brazil. Lepr Rev. 2009;80(2):156-63. PMid:19743619. 